\title{
JMM 2018 LECTURE SAMPLER
}

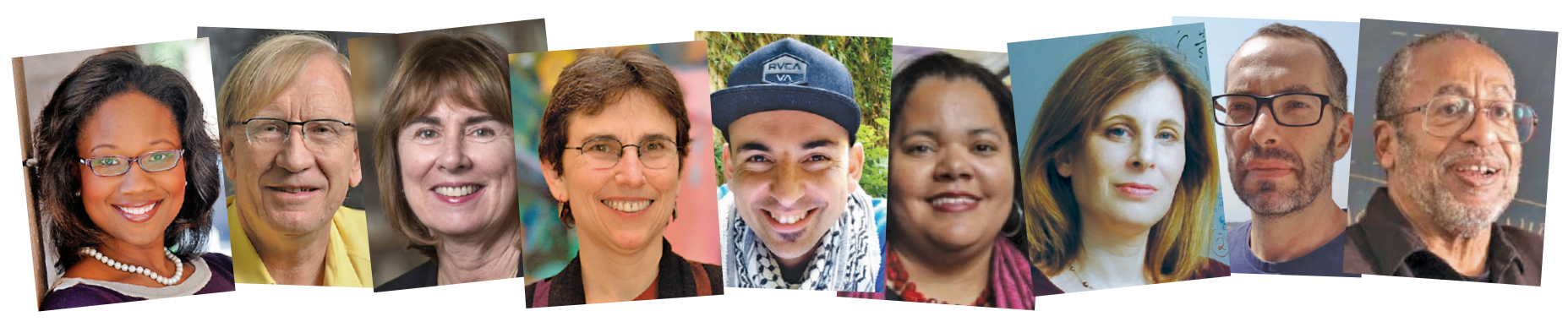

From left to right: Talithia Williams, Gunnar Carlsson, Jill C. Pipher, Ruth Charney, Federico Ardila, Erica Walker, Dana Randall, André Neves, Ronald E. Mickens.

Some of the Joint Mathematics Meetings invited speakers have kindly provided these introductions to their lectures in order to entice meeting attendees and to include non attendees in the excitement.

page 7 Talithia Williams, "Mathematics for the Masses" (AMS-MAA-SIAM Hrabowski-Gates-Tapia-McBay Lecture) 9:00 am-9:50 am, Wednesday, January 10

page 8 Gunnar Carlsson, "Topological Modeling of Complex Data" 11:10 am-12:00 pm, Wednesday, January 10

page 9 Jill C. Pipher, "Nonsmooth Boundary Value Problems" (AWM-AMS Noether Lecture) 10:05 am-10:55 am, Thursday, January 11

page 10 Ruth Charney, "Searching for Hyperbolicity" 3:20 pm-4:10 pm, Thursday, January 11

page 11 Federico Ardila, "Algebraic Structures on Polytopes" 2:15 pm-3:05 pm, Thursday, January 11

page 13 Erica Walker, "Hidden in Plain Sight: Mathematics Teaching and Learning through a Storytelling Lens" 7:45 pm-8:35 pm, Friday, January 12

page 14 Dana Randall, "Emergent Phenomena in Random Structures and Algorithms" 10:05 am-10:55 am, Friday, January 12

page 16 André Neves, "Minimal Surfaces, Volume Spectrum, and Morse Index" (Lecture title: Wow, So Many Minimal Surfaces!)

11:10 am-12:00 pm, Friday, January 12

page 17 Ronald E. Mickens, "Nonstandard Finite Difference Schemes" 1:00 pm-1:50 pm, Saturday, January 13 


\section{Talithia Williams}

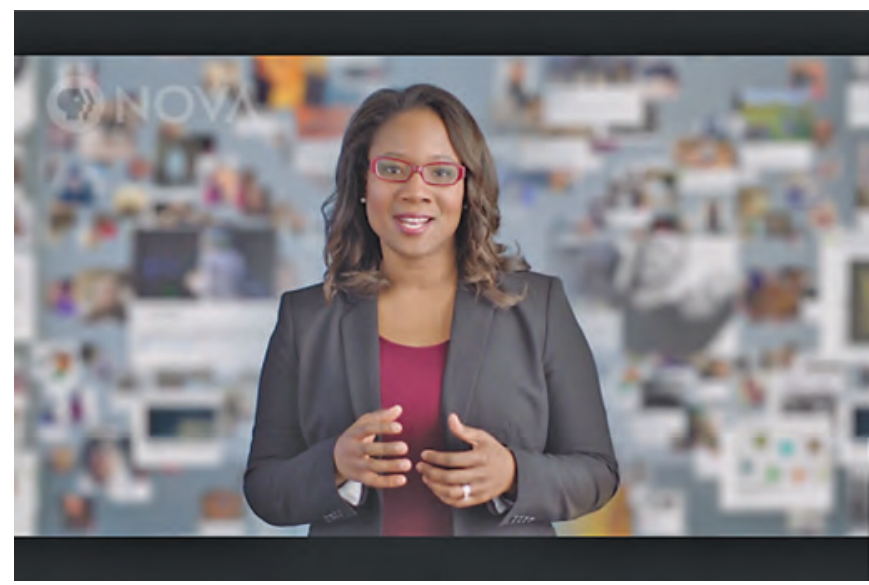

Williams is a host of PBS's new NOVA Wonders series, premiering this spring, Tuesdays at $10 \mathrm{pm}$.

\section{Mathematics for the Masses (AMS-MAA-SIAM Hrabowski-Gates-Tapia-McBay Lecture)}

In recent months, we've witnessed Americans grapple with the significance of science, technology, engineering, and mathematics (STEM) through events ranging from the Paris Agreement to the nationwide March for Science, where people marched to defend the role of science in society. In the wake of a renewed excitement for STEM, I'm thrilled to be joining the Public Broadcasting System (PBS) family as one of the hosts of a new NOVA series called NOVA Wonders, premiering this spring. NOVA is the most-watched prime-time science series on television, reaching an average of five million viewers weekly.

NOVA Wonders is a six-part series that will journey to the frontiers of science, where researchers are tackling some of the most intriguing questions about life and the cosmos. My goal in hosting the show is to open individuals to the power of mathematics and data to pursue answers to questions in a clear and purposeful way. Each hour explores a different big question, from the mysteries of astrophysics to the challenges of inventing technologies that could rival the abilities of the human mind. During this talk, I plan to share with you an early clip from NOVA Wonders, which I have the pleasure of hosting along with neurobiologist André Fenton and computer scientist Rana El-Kaliouby. I'll also discuss ways that we can take our mathematics to the masses and share techniques that

Talithia Williams is associate dean for Research and Experiential Learning and associate professor of mathematics at Harvey Mudd College. Her email address is twi11iams@hmc. edu.

For permission to reprint this article, please contact:

reprint-permission@ams.org.

DOI: http://dx.doi.org/10.1090/noti1616 have been successful in my environment. We all have a responsibility to inspire a new generation in STEM and nurture the dreams of future mathematical leaders.

\section{Image Credits}

Photo from NOVA Wonders (c)2017 WGBH. Author headshot $\subset$ Harvey Mudd College.

ABOUT THE AUTHOR

Talithia Williams researches the spatial and temporal structure of data and environmental applications. She has partnered with the World Health Organization on a cataract surgical rate model for Africa. Her TED talk, "Own Your Body's Data," has over a million views. She is a guest Notices editor for the upcoming February issue for Black History Month.

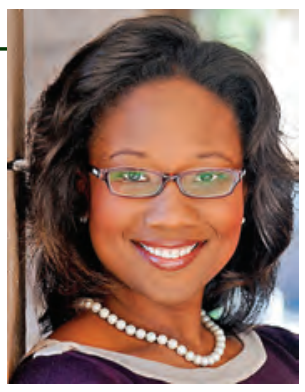

Talithia Williams 


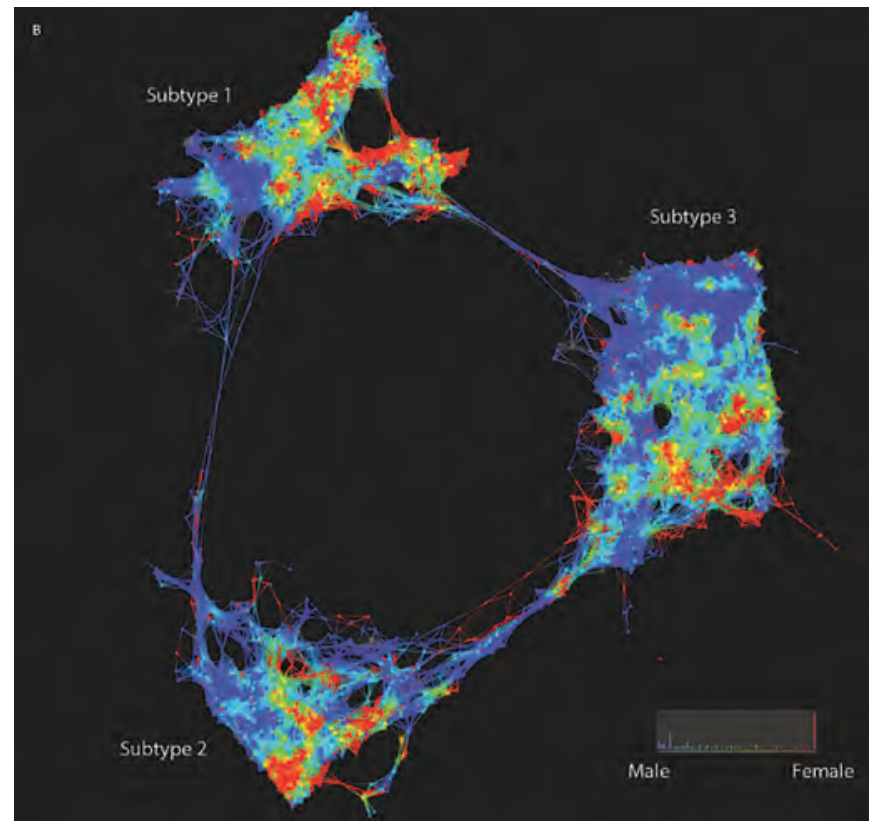

Figure 1. A network model for diabetics shows three distinct types, one heavily correlated with cancer.

\section{Gunnar Carlsson}

\section{Topological Modeling of Complex Data}

In the talk, we will be discussing the modeling of complex data sets by networks. Here are a couple of examples of applications of this idea. The first was a model for a data set of type 2 diabetics constructed by a group at the Mt. Sinai School of Medicine. The data set included genomic data as well as information from electronic medical records. Figure 1 shows a layout of the network that was obtained.

You can see that the network contains three large groups connected by "thin wires." The conclusion the Mt. Sinai researchers were able to draw from this is that type 2 diabetes, rather than being a single disease, is actually made up of three distinct types, and they found that one of these groups was heavily correlated with cancer. The finding that there are three distinct diseases will clearly have implications for treatment of the disease and constitute a contribution to "precision medicine."

A second example comes from the laboratory of David Schneider, a microbiologist at Stanford University. He studies the progression of infectious diseases. He has constructed a number of data sets using both physiological as well as genomic measurements on the subjects. Figure 2 shows an image of a collection of network models

Gunnar Carlsson is professor of mathematics at Stanford University. His email address is car1sson@stanford. edu.

For permission to reprint this article, please contact:

reprint-permission@ams.org.

DOI: http://dx.doi.org/10.1090/noti1618

he constructed for mice and humans infected by flu and malaria.

You will notice that the models are both loops. This reflects the fact that they represent a phenomenon that begins at the healthy state, proceeds through gradual development of the disease until the immune response becomes strong, and then returns to the healthy state.

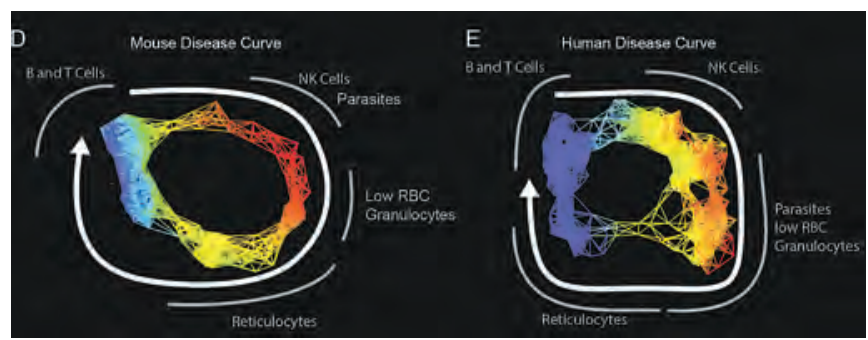

Figure 2. Mouse and human disease network models each form a loop through disease and recovery.

These models provide a time-independent model for the actual state of the subject. It is important to have such a model since (a) the progression of disease occurs at different rates for different subjects and (b) we don't generally have knowledge of the time the infection occurred. This should also be viewed as a contribution to precision medicine.

In the lecture we will discuss these and numerous other examples.

\section{Image Credits}

Figure 1 courtesy of "From identification of type 2 diabetes subgroups through topological analysis of patient similarity," L. Li, W-Y. Cheng , B. Glicksberg, O. Gottesman, R. Tamler, R. Chen, E. Bottinger, and J. Dudley Science Translational Medicine 28 Oct 2015: Vol. 7, Issue 311, pp. 311ra174. DOI:10.1126/scitranslmed.aaa9364.

Figure 2 courtesy of From Tracking Resilience to Infections by Mapping Disease Space, B.Y. Torres, J.H.M. Oliveira, Tate A. Thomas, P. Rath, K. Cumnock, and D.S. Schneider, PLoS Biol. 14(4): e1002436. DOI:10.1371/journal.pbio.1002436

Author photo courtesy of Gunnar Carlsson.

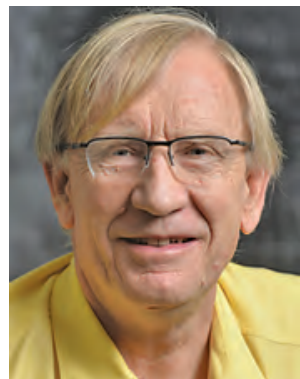

Gunnar Carlsson

\section{ABOUT THE AUTHOR}

Gunnar Carlsson spent most of his career working in the pure aspects of topology, but has turned to applications of the subject in the last fifteen years. 
Jill C. Pipher

\section{Nonsmooth Boundary Value Problems (AWM- AMS Noether Lecture)}

The regularity properties of solutions to linear PDEs in domains in $\mathbb{R}^{n}$ depend on the structure of the equation, the degree of smoothness of the coefficients of the equation, and of the boundary of the domain. Quantifying this dependence is a classical problem, and modern techniques can answer some of these questions with remarkable precision. For both physical and theoretical reasons, it is important to consider partial differential equations with nonsmooth coefficients. We'll discuss how some classical tools in harmonic and complex analysis have played a central role in answering these questions.

In two papers, in 1958 and in 1962, L. Carleson gave a new solution to the problem of interpolation of bounded analytic functions and solved a related problem about the spectrum of bounded holomorphic functions in the disk (the "corona" theorem). The interpolation problem is about finding a bounded analytic function that takes on prescribed values $w_{j}$ at prescribed locations $z_{j}$. Carleson's dual formulation of the interpolation problem led him to the following question:

Let $d \mu$ be a measure in the unit disk. Suppose $G$ is an analytic function in the unit disk with the norm, for $p \geq 1$,

$$
\|G\|_{p}=\lim _{r \rightarrow 1}\left(\frac{1}{2 \pi} \int_{-\pi}^{\pi} \mid G\left(\left.r e^{i \theta}\right|^{p} d \theta\right)^{1 / p} .\right.
$$

Then, under what conditions on $d \mu$ do we have the following inequality:

$$
\int|G(z)|^{p} d \mu(z) \leq C\|G\|_{p}^{p}
$$

Carleson's 1958 paper answered this question for certain point masses, and his 1962 paper gave the general solution. Measures with this property became known as Carleson measures and found their way into many different areas of analysis, including operator theory, the Caldéron-Zygmund theory of singularity integrals, and geometric measure theory, to name a few. The main thrust of this lecture is the crucial role such measures have played in the development of the theory of elliptic and parabolic equations.

Indeed, in exactly the same timeframe of 1958-1962, there were some groundbreaking developments in the theory of divergence form elliptic and parabolic equations, known as the De Giorgi-Nash-Moser theory. The objects of study here are solutions to elliptic equations or parabolic equations with nonsmooth coefficients, broad

Jill C. Pipher is Elisha Benjamin Andrews Professor of Mathematics, Brown University. Her email address is Ji11_Pipher abrown.edu.

For permission to reprint this article, please contact: reprint-permission@ams.org.

DOI: http://dx.doi.org/10.1090/noti1620 generalizations of two classical operators, Laplace's equation and the heat equation. Nash's 1958 paper begins by describing his motivation for the consideration of nonsmooth coefficients. He wanted to understand the behavior of solutions to the nonlinear parabolic equations of flow for a heat-conducting fluid. De Giorgi was led to the same problem of regularity of solutions to elliptic equations with nonsmoooth coefficients in an effort to resolve Hilbert's nineteenth problem, which asks if "the solutions of regular problems in the calculus of variations [are] always necessarily analytic."

The elliptic equations considered by De Giorgi and Nash are in divergence form: $L=\operatorname{div} A(x) \nabla$, where the matrix $A(x)$ has bounded measurable coefficients $a_{i, j}(x)$ and is strongly elliptic. The ellipticity condition means that the spectrum of eigenvalues of $A(x)$ lies between two fixed positive parameters. Observe that when $A$ is the identity matrix, $L$ is just the classical Laplacian, whose solutions are the harmonic functions.

What does it mean to have a solution to such an equation, $\operatorname{div} A(x) \nabla u=0$, when we can't differentiate the coefficients $a_{i, j}(x)$ ? Putting this aside for the moment, we can instead assume that the coefficients $a_{i, j}(x)$ are smooth and ask what estimates on solutions can be obtained that are independent of any quantitative measure of that smoothness. De Giorgi and Nash proved independently that solutions to such divergence form elliptic equations possess a degree of continuity in the interior of the domain that can be measured in terms of the ellipticity parameters alone. This degree (Hölder) of continuity is best possible in such generality. This contrasts sharply with harmonic functions, which are real analytic by virtue of solving Laplace's equation.

In 1961 Moser took a different approach to proving continuity of solutions via a powerful iterative technique. His proof relied on a property of functions of bounded mean oscillation (BMO) that had just been discovered by John and Nirenberg. (In a striking coincidence, their seminal paper on this subject was published in the same issue of the CPAM journal as Moser's paper.) In Moser's use of the John-Nirenberg theory of BMO functions, one finds a surprising connection to Carleson's measures, for it turns out, as C. Fefferman showed ten years later, that BMO functions and Carleson measures are intimately related: the harmonic extension of a bounded or BMO function on $\mathbb{R}^{n-1}$ gives rise to an expression that is a Carleson measure in the upper half-space $\mathbb{R}_{+}^{n}$. This discovery about harmonic functions has found far-reaching extensions in the elliptic theory.

I hope to give the flavor of some results and open problems in this subject at the interface of harmonic analysis and PDE and describe some joint work in recent years with M. Dindos, S. Hofmann, C. Kenig, S. Mayboroda, S. Petermichl, D. Rule, T. Toro, and others. 
Image Credit

Author photo courtesy of Brown University.

\section{ABOUT THE AUTHOR}

Jill C. Pipher was formerly president of the Association for Women in Mathematics and is founding director of the Institute for Computational and Experimental Research in Mathematics. She is currently the American Mathematical Society president-elect.

\section{Ruth Charney}

\section{Searching for Hyperbolicity}

As students, we first encounter groups as algebraic objects, but groups can also be viewed as symmetries of geometric objects. This viewpoint gives rise to powerful tools for studying infinite groups. The work of Max Dehn in the early 1900s on groups acting on the hyperbolic plane was an early indication of this phenomenon. Dehn's ideas were vastly generalized in the 1980s by Cannon and Gromov to a large class of groups, known as Gromov hyperbolic groups. In recent years there has been an effort to push these ideas even further. If a group fails to be Gromov hyperbolic, might it still display some hyperbolic behavior? Might some of the techniques used in hyperbolic geometry still apply?

This talk will begin with an introduction to some basic ideas in geometric group theory, including Gromov's notion of hyperbolicity, and conclude with a discussion of recent work on finding and encoding hyperbolic behavior in more general groups. A version of this talk was given at the AWM Research Symposium 2017, and a more extended abstract can be found in the "Lecture Sampler" in the April 2017 Notices. $^{1}$

\section{Image Credit}

Author photo courtesy of Michael Lovett.

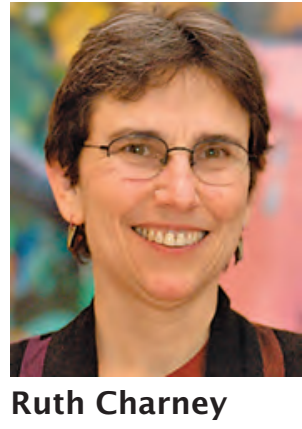

ABOUT THE AUTHOR

Ruth Charney's research focuses on the interplay between groups and geometry. She has also worked extensively with professional organizations, serving as vice-president and trustee of AMS and as president of AWM.

Ruth Charney is Theodore and Evelyn Berenson Professor of Mathematics at Brandeis University. Her email address is charney@ brandeis. edu.

1 www.ams.org/pub1ications/journals/notices/201704 /rnoti-p341.pdf

For permission to reprint this article, please contact: reprint-permission@ams . org.

DOI: http://dx.doi.org/10.1090/noti1623 


\section{Federico Ardila}

\section{Algebraic Structures on Polytopes}

AUTHOR'S NOTE. My talk will discuss the algebraic and combinatorial structure of a beautiful family of polytopes. It is based on joint work with Marcelo Aguiar. It will be accessible to undergraduates and will not assume any previous knowledge of these topics.

\section{Two Classical Problems: Inverting Power Series} Multiplicative Inversion

Let us consider two power series $A(x)=1+\sum_{n \geq 1} a_{n} \frac{x^{n}}{n !}$ and $B(x)=1+\sum_{n \geq 1} b_{n} \frac{x^{n}}{n !}$ that are multiplicative inverses; i.e., $A(x) B(x)=1$. The first few coefficients of $B(x)=1 / A(x)$ are

$$
\begin{aligned}
& b_{1}=-a_{1}, \\
& b_{2}=-a_{2}+2 a_{1}^{2}, \\
& b_{3}=-a_{3}+6 a_{2} a_{1}-6 a_{1}^{3}, \\
& b_{4}=-a_{4}+8 a_{3} a_{1}+6 a_{2}^{2}-36 a_{2} a_{1}^{2}+24 a_{1}^{4} .
\end{aligned}
$$

\section{Compositional Inversion}

Consider two power series $C(x)=x+\sum_{n \geq 2} c_{n-1} x^{n}$, and $D(x)=x+\sum_{n \geq 2} d_{n-1} x^{n}$ that are compositional inverses; i.e., $C(D(x))=x$. The first few coefficients of $D(x)=$ $C(x)^{\langle-1\rangle}$ are

$$
\begin{aligned}
& d_{1}=-c_{1}, \\
& d_{2}=-c_{2}+2 c_{1}^{2}, \\
& d_{3}=-c_{3}+5 c_{2} c_{1}-5 c_{1}^{3}, \\
& d_{4}=-c_{4}+6 c_{3} c_{1}+3 c_{2}^{2}-21 c_{2} c_{1}^{2}+14 c_{1}^{4} .
\end{aligned}
$$

It is natural to ask: What do these coefficients count? Two families of polytopes hold the answers.


Figure 1 . The permutahedra $\pi_{1}, \pi_{2}, \pi_{3}, \pi_{4}$ whose face structures tell us how to compute the multiplicative inverse of a series.

Federico Ardila is professor of mathematics, San Francisco State University, and Simons Professor, Mathematical Sciences Research Institute. His email address is federico@sfsu.edu.

Supported by NSF CAREER grant DMS-0956178 and Combinatorics grants DMS-0801075, DMS-1440140, and DMS-1600609.

For permission to reprint this article, please contact: reprint-permission@ams .org.

DOI: http://dx.doi.org/10.1090/noti1621
The permutahedron $\pi_{n}$ (see Figure 1) is the polytope in $\mathbb{R}^{n}$ whose vertices are the $n$ ! permutations of $\{1,2, \ldots, n\}$, regarded as vectors. Every face of a permutahedron is a product of permutahedra of lower dimensions.

The face structure of permutahedra tells us how to compute $B(x)=1 / A(x)$. For example, the formula for $b_{4}$ shown above is determined by the faces of $\pi_{4}$ :

- 1 truncated octahedron $\pi_{4}$,

- 8 hexagons $\pi_{3} \times \pi_{1}$ and 6 squares $\pi_{2} \times \pi_{2}$,

- 36 segments $\pi_{2} \times \pi_{1} \times \pi_{1}$, and

- 24 points $\pi_{1} \times \pi_{1} \times \pi_{1} \times \pi_{1}$.

The signs are given by the dimensions of the faces.
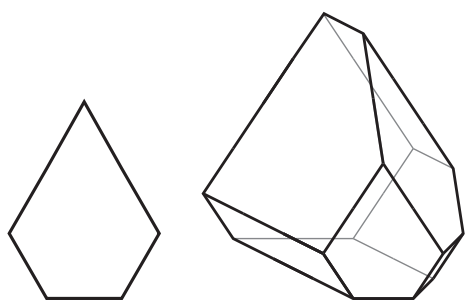

Figure 2. The associahedra $\mathfrak{a}_{1}, \mathfrak{a}_{2}, \mathfrak{a}_{3}, \mathfrak{a}_{4}$ whose face structures tell us how to compute the compositional inverse of a series.

The associahedron $\mathfrak{a}_{n}$ (see Figure 2) is a polytope in $\mathbb{R}^{n}$ whose vertices correspond to the $C_{n}=\frac{1}{n+1}\left(\begin{array}{c}2 n \\ n\end{array}\right)$ associations of a product $x_{1} \cdots x_{n}$ into binary products. For example, when $n=5$, one such association is $\left(x_{1} X_{2}\right)\left(\left(x_{3} X_{4}\right) x_{5}\right)$. Every face of an associahedron is a product of associahedra of smaller dimensions.

The face structure of permutahedra tells us how to compute $D(x)=C(x)^{\langle-1\rangle}$. For instance, the formula for $d_{4}$ shown above comes from the faces of $\mathfrak{a}_{4}$ :

- 1 three-dimensional associahedron $\mathfrak{a}_{4}$,

- 6 pentagons $\mathfrak{a}_{3} \times \mathfrak{a}_{1}$ and 3 squares $\mathfrak{a}_{2} \times \mathfrak{a}_{2}$,

- 21 segments $\mathfrak{a}_{2} \times \mathfrak{a}_{1} \times \mathfrak{a}_{1}$, and

- 14 points $\mathfrak{a}_{1} \times \mathfrak{a}_{1} \times \mathfrak{a}_{1} \times \mathfrak{a}_{1}$.

Again, the signs come from the face dimensions.

We discovered these results unexpectedly when studying a more general family of polytopes.

\section{Hopf Monoids and Generalized Permutahedra}

Edmonds, Stanley, and others taught us that to study combinatorial objects, it is often helpful to build polyhedral models for them. Generalized permutahedra (or equivalently submodular functions) are a particularly useful family of polyhedra. They are deformations of the permutahedron, obtained by moving the faces while preserving their directions. Two important families are the permutahedra and associahedra, but there are many more. Figure 3 shows a few three-dimensional examples.

Joni and Rota, Joyal, Stanley, and others showed that to study combinatorial objects, it is often helpful to endow them with algebraic structures. In their book Monoidal Functors, Species and Hopf Algebras (2010), Aguiar and Mahajan provide a particularly useful framework: Hopf 

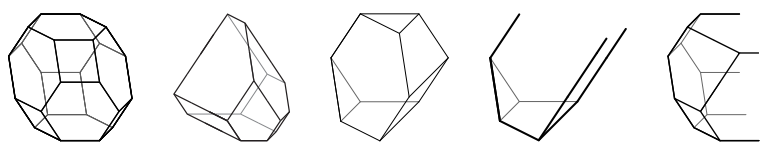

Figure 3. Five generalized permutahedra.

monoids in species. This framework applies to families of combinatorial objects having natural operations of merging two disjoint objects into one and breaking an object into two disjoint parts, subject to various axioms.

Many families of combinatorial objects carry such polyhedral and algebraic structures: graphs, matroids, posets, set partitions, and simplicial complexes, to name just a few. The main idea of this project is to bring together these two points of view:

Theorem 1. Generalized permutahedra form a Hopf monoid in species GP. In fact, they are the universal family of polyhedra with this algebraic structure. The Hopf monoid GP contains or projects to the Hopf monoids G, M, P, П, SC of graphs, matroids, posets, set partitions, and simplicial complexes.

\section{Combinatorial Hopf Monoids and Antipodes}

A key component of a Hopf monoid is its antipode map $S$, which is analogous to the inverse map in a group. The antipode is given by a very large alternating sum, generally involving lots of cancellation. A fundamental and often difficult question is to compute this antipode. Figure 4 shows the antipode of a graph and of a poset.

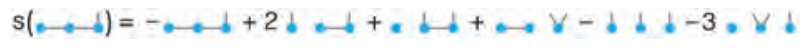

$s(X)=-X+2 \Lambda \cdot+2 \bigvee \cdot-4 \jmath \cdot \cdots \cdot$

Figure 4. The antipode of a graph and of a poset.

These formulas result from simplifying alternating sums of 13 and 75 terms, respectively. How can we systematically explain the extensive cancellation that is taking place?

This is a rather subtle question. For instance, although most of the Hopf algebraic structures G, M, P, П, SC have been known for decades, optimal formulas for their antipodes were not known until very recently.

Fortunately, generalized permutahedra provide a geometric setting where the cancellation may be completely understood topologically. This allows us to obtain the optimal formula for the antipode of generalized permutahedra.

Theorem 2. The antipode of the Hopf monoid GP is given by the following cancellation-free and grouping-free formula: If $\wp$ is a generalized permutahedron in $\mathbb{R}^{n}$, then

$$
\mathrm{S}(\wp)=\sum_{\mathfrak{q} \text { face of } \wp}(-1)^{n-\operatorname{dim} \mathfrak{q}} \mathfrak{q} .
$$

This automatically yields the optimal formulas for the antipodes of $\mathrm{G}, \mathrm{M}, \mathrm{P}, \Pi$, and $\mathrm{SC}$.

\section{Inverting Formal Power Series, Revisited}

Once we discovered these results, we asked: What happens when we apply the general theory of the Hopf monoid $G P$ to the special families of polytopes $\pi_{1}, \pi_{2}, \pi_{3}, \ldots$ and $\mathfrak{a}_{1}, \mathfrak{a}_{2}, \mathfrak{a}_{3}, \ldots$ ? The answer was very surprising to me: Theorem 2 implies that the multiplicative and compositional inverses of power series are given by the alternating sum of the faces of permutahedra and associahedra, as explained above.

\section{Image Credits}

Figures courtesy of Federico Ardila. Author photo courtesy of May-Li Khoe.

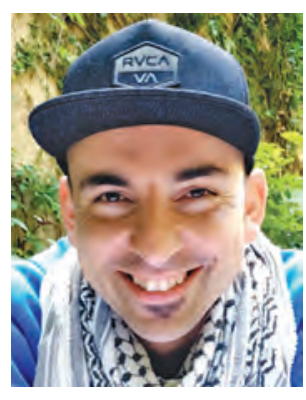

Federico Ardila

\section{ABOUT THE AUTHOR}

Federico Ardila works in combinatorics and its connections to other areas of mathematics and applications. He also strives to build joyful and empowering mathematical spaces. He has advised forty thesis students in the US and Colombia; among his US students, more than half are members of underrepresented groups and more than half are women. Outside of work, he is often playing fútbol or DJing with his wife, May-Li, and Colectivo La Pelanga. 


\section{Erica Walker}

\section{Hidden in Plain Sight: Mathematics Teaching and Learning through a Storytelling Lens}

Our stories about mathematical excellence are too often hidden. The recent movie Hidden Figures, surrounding Katherine Johnson and her colleagues for their work at NASA, is the kind of story about mathematics that can inform us how to make mathematics learning experiences-in and out of school-more diverse, rewarding, engaging, transformative, rigorous, and commonplace. Such stories can help develop cadres of young people with strong and positive mathematics identities who are excited about mathematics; who see themselves and are seen as talented, knowledgeable doers and users of mathematics; and who are leaders of robust learning communities. Our work as mathematicians and educators must include uncovering the hidden and making known the unknown. How can we use narratives of excellence to address the many leaky junctures within the school mathematics pipeline: more negative attitudes towards math the longer students are in school; disparities in course-taking critical for college access and completion; fewer and fewer students interested in pursuing mathematics related careers? And how can we learn from the use of narratives in other disciplines to promote mathematics engagement and competence in the public sphere? My research suggests that there is much to be learned from the stories that mathematicians tell about the people and places that were critical to their mathematics development. And these stories can inspire teachers and students and generate public interest in mathematics and mathematicians. The narratives and themes shared in this talk will reveal successful strategies using hidden stories in the service of the human and mathematical development of all.

\author{
Image Credit
}

Author photo courtesy of Bruce Gilbert.

ABOUT THE AUTHOR

Erica Walker's research explores sociological and cultural factors influencing participation and performance in mathematics. She has published in many journals and is the author of two books, one of which is a study of mathematicians in the United States (Beyond Banneker: Black Mathematicians and the Paths to Excellence, published by SUNY Press). A museum explorer and inveterate stroller, she is fond of finding mathematics in everyday and unusual spaces.
Erica Walker is professor of mathematics education at Teachers College, Columbia University. Her email address is ewa7ker@tc . edu.

For permission to reprint this article, please contact:

reprint-permission@ams.org.

DOI: http://dx.doi.org/10.1090/noti1615



Erica Walker 


\section{JMM 2018 LECTURE SAMPLER}

\section{Dana Randall}

\section{Emergent Phenomena in Random Structures and Algorithms}

Monte Carlo methods have become ubiquitous across science and engineering to model dynamics and explore large sets of configurations. The idea is to perform a random walk among the allowable configurations so that even though only a very small part of the space is visited, samples will be drawn from close to a desirable distribution, enabling statistical inferences. Over the last twenty-five years there have been tremendous advances in the design and analysis of Markov chains for this purpose, building on insights from physics, discrete probability, and theoretical computer science. The main objectives include designing provably efficient sampling algorithms, finding computational problems amenable to this approach, and developing new mathematical tools for bounding convergence times for these stochastic processes.

One of the striking discoveries has been the realization that many natural Markov chains undergo a phase transition, whereby they abruptly change from being efficient (and usable) to being inefficient (and thereby impractical for large problems) as some parameter of the system is varied. Figure 1 shows an example in which the particles align only above a critical density. Another example is the following chain that samples from the set of independent sets of a graph, known in the statistical physics community as the hard-core lattice gas model. We are given a graph $G$ and a parameter $\lambda>0$, known as the activity (or fugacity). The state space $\Omega$ is the set of independent sets of $G$ and our goal is to sample from the Gibbs (or Boltzmann) distribution

$$
\pi(I)=\lambda^{|I|} / Z,
$$

where $I \in \Omega$ is an independent set, $|I|$ is its size, and $Z=\sum_{J \in \Omega} \lambda^{|J|}$ is the normalizing constant known as the partition function. When $\lambda>1$ we are favoring dense independent sets, and when $\lambda<1$ we are favoring sparse ones. Local dynamics can be defined so that we can move between pairs of configurations with Hamming distance one, i.e., independent sets that differ by the addition or deletion of a single vertex, or we can stay where we are. The celebrated Metropolis algorithm tells us the probabilities with which to implement these transitions so that iterating the moves for sufficiently long will generate samples from close to the target distribution $\pi$. Starting

Dana Randall is professor of computer science and adjunct professor of mathematics at the Georgia Institute of Technology. Her email address is randa11@cc.gatech.edu.

This work was supported in part by NSF CCF-1526900 and CCF1637031.

For permission to reprint this article, please contact: reprint-permission@ams.org.

DOI: http://dx.doi.org/10.1090/noti1622



Figure 1. A discrete model for programmable active matter where particles align only above a critical high density.

at any configuration $\sigma \in \Omega$, say the empty independent set (with no vertices), we repeat the following: choose a vertex $v$ at random; if $v$ is in the current independent set, remove it with probability $\min \left(1, \lambda^{-1}\right) / 2$; if it is not in the independent set, add it with probability $\min (1, \lambda) / 2$ if doing so does not violate the independence requirement; in all other cases, keep the independent set unchanged. It is simple to show that this chain connects the state space and converges to the unique stationary distribution $\pi$, so our goal is to determine whether it converges quickly enough to be practical.

It turns out that for small values of $\lambda$, the Metropolis chain on independent sets converges quickly to stationarity and provides an efficient way to sample, while for large values of $\lambda$ it is prohibitively slow. To see why, imagine the underlying graph $G$ is an $n \times n$ region of $\mathbb{Z}^{2}$. Dense independent sets dominate the stationary distribution $\pi$ when $\lambda$ is large, and it will take exponential time (in $n$ ) to move from an independent set that lies mostly on the odd sublattice to one that is mostly even.

This type of dichotomy is well known in the statistical physics community, where many models have been shown to abruptly transition from a disordered state to a predominantly ordered one, characterized by some emergent phenomenon. Physicists observe phase transitions when determining whether there is a unique limiting Gibbs measure on the infinite lattice, known as a Gibbs state. For the hard-core model on $\mathbb{Z}^{2}$, it is believed that there exists a critical value $\lambda_{c}$ such that for $\lambda<\lambda_{c}$ there is a unique Gibbs state, while for $\lambda>\lambda_{c}$ there are multiple Gibbs 


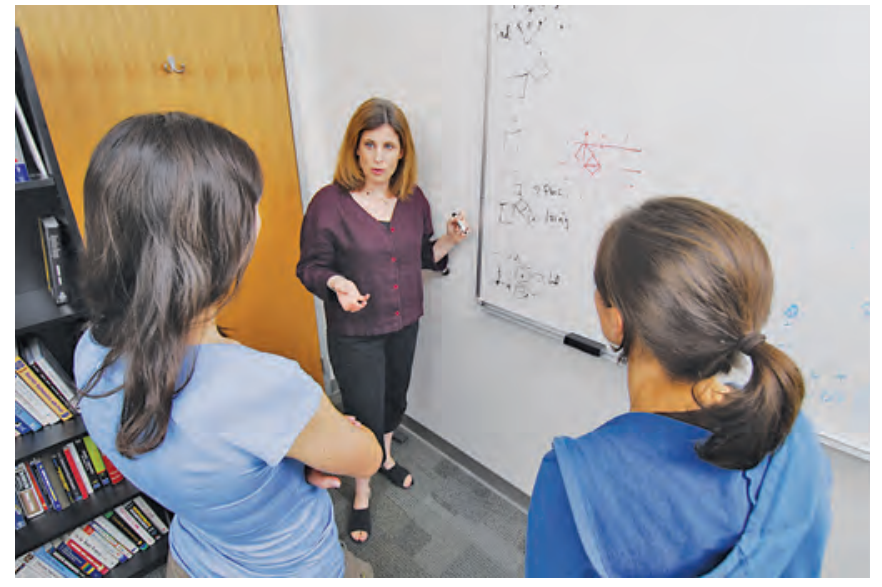

Figure 2. Randall working with former PhD students Amanda Pascoe Streib and Sarah Miracle.

states. This has been verified for small and large values of $\lambda$ bounded away from the conjectured critical point $\lambda_{c} \approx 3.79$ in both the computational and the physics settings [1], [2].

We will explore how phase transitions in random structures and algorithms can provide valuable insights in three contexts. First, they allow us to understand the efficacy and limitations of certain classes of sampling algorithms, potentially leading to faster alternative approaches. Second, they reveal statistical properties of stationary distributions, giving insight into various interacting models from across the sciences. Examples include colloids or binary mixtures of molecules in suspension; segregation models, where individuals are more likely to move when they are dissatisfied with their local demographics; and interacting particle systems from statistical physics. Last, we can harness emergent phenomena due to phase transitions as a new algorithmic tool in order to coordinate behavior in various asynchronous distributed systems. Examples include models of programmable active matter and swarm robotics. We will see how these three research threads are closely interrelated, informing one another in surprising ways.

\section{References}

[1] A. Blanca, Y. Chen, D. Galvin, D. RAndall, and P. Tetali, Phase coexistence for the hard-core model on $\mathbb{Z}^{2}$, preprint, arXiv:1611.01115, 2016.

[2] J. C. VERA, E. VIGODA, and L. YANG, Improved bounds on the phase transition for the hard-core model in 2-dimensions, in P. Raghavendra, S. Raskhodnikova, K. Jansen, J. D. P. Rolim (eds), Approximation, Randomization, and Combinatorial Optimization. Algorithms and Techniques, Lecture Notes in Comput. Sci., vol. 8096, Springer, Heidelberg, 2013. MR3126563

\section{Image Credits}

Figure 1 courtesy of Shengkai Li, School of Physics, Georgia Tech. Figure 2 courtesy of Georgia Tech College of Computing. Author photo courtesy of Georgia Tech College of Computing.

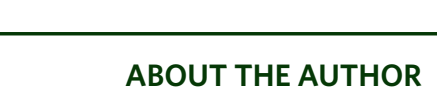

Dana Randall studies randomized algorithms at the interface between theoretical computer science, discrete probability, and statistical physics. Last year she became co-executive director of the Institute for Data Engineering and Science, an interdisciplinary research institute at Georgia Tech that she co-founded. She also serves as the ADVANCE Professor of Computing as an advocate for equity and inclusion. 


\section{André Neves}

\section{Minimal Surfaces, Volume Spectrum, and Morse Index}

A recurrent question in mathematics consists in finding critical points for a given functional defined on some space. Typical questions ask to

(i) characterize the most stable critical points, i.e., those that for all but a small number of perturbations still persist;

(ii) count the number of critical points;

(iii) in case there are infinitely many critical points, study their asymptotic behavior as they become increasingly more unstable.

To give a concrete example, one can consider the space of all closed hypersurfaces in a Riemannian $n+1$-manifold $M$ and the functional that computes the area of these hypersurfaces. Such spaces are well studied in geometric measure theory, a field pioneered by Federer and Fleming in the 1960s. The critical points in this case are called minimal surfaces and are ubiquitous not only in geometry but in may other branches of mathematics as well.

For this particular case, there have been new and exciting developments regarding the questions outlined above, namely:

(i) jointly with Fernando Marqués, the most stable minimal surfaces in the round 3-sphere were classified and used to solve the Willmore Conjecture;

(ii) infinitely many minimal surfaces $\Sigma_{k}$ have been found for a large class of Riemannian manifolds [3], [5] (those with positive Ricci curvature), and their area plus their degrees of instability have been shown to be increasingly large [4];

(iii) the area of these infinitely many minimal surfaces obey a Weyl asymptotic law [2]. More precisely, the sequence $\frac{\operatorname{area}\left(\sum_{k}\right)}{k^{1 /(n+1)}}$ converges, as $k$ tends to infinity, to a universal constant (depending only on $n$ ) multiplied by $(\operatorname{Vol} M)^{n /(n+1)}$.

We end this brief introduction by noting that the sequence area $\left(\Sigma_{k}\right)$ is called the volume spectrum, and a better understanding of its properties would provide answers to other open problems in geometry. For instance, Irie, Marqués, and myself [1], used the Weyl Law to show that for a generic metric, not only are there infinitely many minimal hypersurfaces, but they are dense. In particular this answered a long-standing conjecture of Yau.

\section{References}

[1] K. IRIE, F. C. MARQUÉs, and A. NEVES, Denseness of minimal hypersurfaces for generic metrics, arXiv:1710.10752 [math.DG] (2017).

André Neves is professor of mathematics at the University of Chicago. His email address is aneves@math. uchicago.edu.

For permission to reprint this article, please contact:

reprint-permission@ams.org.

DOI: http://dx.doi.org/10.1090/noti1619

[2] Y. Liokumovich, F. C. MARQuÉs, and A. Neves, Weyl law for the volume spectrum, arXiv:1607.08721 [math.DG], 2016.

[3] F. C. MARQUÉS and A. NEVES, Existence of infinitely many minimal hypersurfaces in positive Ricci curvature, Invent. Math. 209 (2017), 577-616. MR3674223

[4] N. AIEX, Noncompactness of the space of minimal hypersurfaces arXiv:1601.01049 [math.DG], 2016.

[5] X. ZHOU, Min-max hypersurface in manifold of positive Ricci curvature, J. Differential Geom. 105 (2017), 291-343. MR3606731

\section{Image Credit}

Author photo courtesy of André Neves.

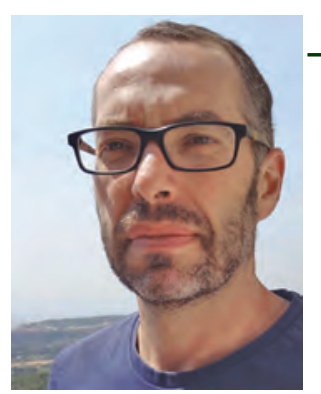

André Neves

\section{ABOUT THE AUTHOR}

André Neves is a geometer, whose goal is to understand which restrictions curvature imposes on space or on its submanifolds. With Fernando Marqués, he proved that the least bended torus in space is the Clifford torus and, in doing so, solved the Willmore conjecture. 


\section{Ronald E. Mickens}

\section{Nonstandard Finite Difference Schemes}

\section{Prelude}

Nonstandard finite difference (NSFD) schemes $^{1}$ are an alternative methodology for constructing discretizations of differential equations for the purpose of calculating numerical solutions. This technique arose from earlier attempts to formulate for classical mechanics a structure based on discrete time rather than the assumption of a continuous time-independent variable [1]. This formalism is consistent with the fact that most differential equations are models for realizable physical phenomena, and the data on these systems are obtained at discrete times. However, since these differential equations cannot be solved in terms of a finite combination of elementary functions, there is a fundamental need to determine numerical approximations to their solutions.

Standard methods based on finite difference discretizations are generally based on mathematical considerations of consistency, convergence, and stability. These issues arise to help in the prevention of numerical instabilities (NI), i.e., the existence of solutions to the finite difference equations which do not correspond to any solutions of the differential equations.

The NSFD methodology is a "physical" based procedure, in the sense that it incorporates into the discretizations many of the important features of the differential equations and/or their solutions.

\section{Exact Schemes}

The genesis of our NSFD methodology is based on the realization of the existence of exact finite difference representations for certain classes of differential equations. To see this, consider the scalar ordinary differential equation

$$
\frac{d x}{d t}=f(x, t, \lambda), \quad x\left(t_{0}\right)=x_{0},
$$

where $f(\cdots)$ has properties such that a unique solution exists and $\lambda$ denotes the set of parameters characterizing the system modeled. Let $F$ denote this solution of Eq. (1), i.e.,

$$
x(t)=F\left(x_{0}, t_{0}, t, \lambda\right) .
$$

Now let the following be a finite difference discretization of Eq. (1) etc.:

$$
x_{k+1}=G\left(x_{0}, k, h, \lambda\right),
$$

Ronald E. Mickens is the Distinguished Fuller E. Calloway Professor of Physics at Clark Atlanta University. His email address is rmickens@cau.edu.

${ }^{1}$ unrelated to nonstandard analysis

For permission to reprint this article, please contact: reprint-permission@ams.org.

DOI: http://dx.doi.org/10.1090/noti1617 where $h=\Delta t, t_{k}=h k, x_{k}=x\left(t_{k}\right)$. The expression in Eq. (3) is an exact finite difference scheme if

$$
x_{k}=x\left(t_{k}\right), \quad h>0 .
$$

For this to occur, we must have

$$
G\left(x_{0}, k, h, \lambda\right)=F\left(x_{k}, t_{k}, t_{k+1}, \lambda\right) ;
$$

i.e., the exact finite difference scheme for Eq. (1) is

$$
x_{k+1}=F\left(x_{k}, t_{k}, t_{k+1}, \lambda\right) .
$$

Our work on the construction of the NSFD methodology was motivated by Eq. (6); i.e., if the general solution to Eq. (1) is known, then from it, its exact finite difference scheme can be constructed.

\section{Examples of Exact Scheme}

The following three elementary equations and their exact schemes clearly illustrate the nonstandard features of these constructions in comparison with the standard discretizations:

$$
\begin{gathered}
\frac{d x}{d t}=\lambda_{1} x-\lambda_{2} x^{2} \rightarrow \frac{x_{k+1}-x_{k}}{\left(e^{\lambda_{1} h}-1\right) / \lambda_{1}}=\lambda_{1} x_{k}-\lambda_{2} x_{k+1} x_{k}, \\
\frac{d^{2} x}{d t^{2}}+\Omega^{2} x=0 \rightarrow \frac{x_{k+1}-2 x_{k}+x_{k-1}}{\left(\frac{4}{\Omega^{2}}\right)\left[\sin \left(\frac{\Omega h}{2}\right)\right]^{2}}+\Omega^{2} x_{k}=0, \\
\frac{d^{2} x}{d t^{2}}=\lambda \frac{d x}{d t} \rightarrow \frac{x_{k+1}-2 x_{k}+x_{k-1}}{\left(e^{\lambda h}-1\right) h / \lambda}=\lambda\left(\frac{x_{k}-x_{k-1}}{h}\right) .
\end{gathered}
$$

\section{NSFD Methodology}

In the early 1970 s we began to analytically derive exact discretization schemes for hundreds of both linear and nonlinear differential equations. From analyses of these results, the general rules for the NSFD methodology emerged. The two most significant rules are: (i) the requirement of more complex mathematical structures for the discrete representations of derivatives and (ii) the nonlocal representation of functions.

In more detail, we have

$$
\frac{d x}{d t} \rightarrow \frac{x_{k+1}-x_{k}}{\phi}, \quad \phi=h+O\left(h^{2}\right),
$$

where $\phi$ is called a denominator function and, in general, is not the simple $h$ used in most of the standard procedures. For particular classes of differential equations, there exists a procedure for calculating $\phi$. Note that $\phi$ depends not only on the step-size, $h=\Delta t$, but also on one or more parameters appearing in the differential equations.

The nonlocal representation of functions means that these terms have, in general, discretizations spread over more than one grid point. The following are some 
examples of these possibilities:

(8a)

$x=2 x-x \rightarrow 2 x_{k}-x_{k+1}, 1$ st-order ODE,

$$
\begin{aligned}
& x^{2} \rightarrow\left\{\begin{array}{l}
x_{k+1} x_{k}, 1 \text { st-order ODE, } \\
\left(\frac{x_{k+1}+x_{k}+x_{k-1}}{3}\right) x_{k}, \text { 2nd-order ODE, }
\end{array}\right. \\
& x^{3} \rightarrow\left(\frac{x_{k+1}+x_{k-1}}{2}\right) x_{k}^{2}, \text { 2nd-order ODE. }
\end{aligned}
$$

The two major techniques to implement the NSFD methodology as applied to particular differential equations are (a) the principle of dynamic consistency (PDC) and (b) the method of partial equations (MPE).

PDC centers on incorporating some property satisfied by the differential equations and/or their solutions into the NSFD scheme. Such properties may include a positivity condition, special solutions such as fixed-points, particular asymptotic properties, etc.

MPE breaks a differential equation into three or more parts of subequations, determines the exact finite difference scheme for each subequation, and then combines them into an overall consistent, single discretization for the original differential equation.

\section{Examples of NSFD Schemes}

The initial value problem

$$
\frac{d x}{d t}=-\lambda \sqrt{x}, \quad x(0)=x_{0}>0
$$

can be discretized via the NSFD scheme

(10)

$$
\frac{x_{k+1}-x_{k}}{h}=-\lambda\left(\frac{x_{k+1}}{\sqrt{x_{k}}}\right) \text { or } \frac{x_{k+1}-x_{k}}{h}=-\lambda\left(\frac{\sqrt{x_{k+1}}+\sqrt{x_{k}}}{2}\right) \text {. }
$$

Similarly, the equation for a simple combustion model

$$
u_{t}=u_{x x}+u^{2}(1-u), \quad 0 \leq u(x, 0) \leq 1,
$$

has an NSFD scheme

$$
\begin{aligned}
& \frac{u_{m}^{k+1}-u_{m}^{k}}{\Delta t}=\left(u_{m+1}^{k}\right)^{2}+\left(u_{m-1}^{k}\right)^{2}-\left(\frac{u_{m+1}^{k}+u_{m-1}^{k}}{2}\right) u_{m}^{k+1} \\
& -\left[\frac{\left(u_{m+1}^{k}\right)^{2}+\left(u_{m-1}^{k}\right)^{2}}{2}\right] u_{m}^{k+1}+\frac{u_{m+1}^{k}-2 u_{m}^{k}+u_{m-1}^{k}}{(\Delta x)^{2}},
\end{aligned}
$$

where $\left.t_{k}=(\Delta t) k\right) x_{m}=(\Delta x) m$, and $u_{m}^{k} \approx u\left(x_{m}, t_{k}\right)$.

\section{Comments}

The NSFD methodology has been applied to a broad range of differential equations modeling a diverse set of physical phenomena which include:

- interacting populations,

- nonlinear heat transport,

- impulsive systems,

- delay equations,

- equations having fractional derivatives,

- computational electromagnetics,

- singular perturbation problems, and

- simulation of robotic systems.

A review paper summarizing such applications is the recent publication by Patidar [3].

An important insight coming from an analysis of the NSFD methdology is that a proper or valid discretization of a differential equation involves a deep understanding of the whole equation. This is in opposition to standard discretization methods which are based on constructing discrete models of the individual terms appearing in the differential equation and then adding them together to form the final full discretization. One consequence of this fact is that black-box, "plug-in algorithms," which are implemented for standard numerical integration techniques, do not exist for the NSFD methodology.

We welcome you to come to our talk, where a more thorough and deeper discussion of the above issues will be given. This will also allow you to ask interesting and thoughtful questions related to the construction and use of the NSFD philosophy to discretize differential equations.

\section{References}

[1] D. GreEnspan, Discrete Models, Addison-Wesley, Reading, MA, 1973. MR0366132

[2] R. E. MickENS, Nonstandard Finite Difference Models of Differential Equations, World Scientific, River Edge, NJ, 1994. MR1275372

[3] K. C. PATIDAR, Nonstandard finite difference methods: Recent trends and further development, J. of Difference Equ. Appl. 22 (2016), 817-849. MR3520261

\section{Image Credit}

Author photo courtesy of Ronald E. Mickens.

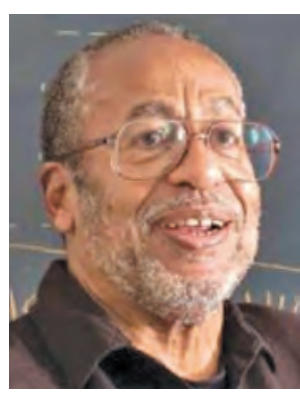

Ronald E. Mickens

\section{ABOUT THE AUTHOR}

Ronald E. Mickens's research interests include asymptotics of difference equations, numerical analysis, mathematical modeling of interacting populations, nonlinear oscillations, and the history and philosophy of science. He is also involved in a variety of mentoring activities for students and junior faculty. 